The Astrophysical JodRnaL, 215:L53-L55, 1977 July 15

(c) 1977. The American Astronomical Society. All rights reserved. Printed in U.S.A.

\title{
NUCLEAR $\gamma$-RAY LINES IN ACCRETION SOURCE SPECTRA
}

\author{
J. C. Higdon \\ Cosmic Ray Working Group, Huygens Laboratory, Leiden, The Netherlands \\ AND \\ R. E. LINGENFELTER* \\ Departments of Astronomy and Earth and Space Sciences, University of California, Los Angeles \\ Received 1977 March 7; revised 1977 A pril 28
}

\begin{abstract}
Gamma-ray line emission is calculated for thermonuclear excitation of very high-temperature $\left(10^{10}-10^{12} \mathrm{~K}\right)$ ions which may exist in accreting matter around neutron stars and black holes. The $4.438 \mathrm{MeV}$ line from ${ }^{12} \mathrm{C}^{*}$ de-excitation should be the easiest to observe in both the continuous and transient emission spectra of such sources.

Subject headings: black holes — gamma rays: general — stars: neutron - X-rays: sources
\end{abstract}

Models of X-and $\gamma$-ray emission from accreting neutron stars (Shapiro and Salpeter 1975) and black holes (Shapiro, Lightman, and Eardley 1976) involve transferring the gravitational energy of infalling ions, which can have temperatures of $\geq 10^{11} \mathrm{~K}$, to cooler $\sim 10^{9} \mathrm{~K}$ electrons which radiate the energy as X-and $\gamma$-rays by bremsstrahlung and Compton scattering. In this Letter we consider direct $\gamma$-ray line emission from the ions themselves, resulting from thermonuclear excitation. Ramaty, Börner, and Cohen (1973) estimated such line emission in connection with positron annihilation radiation for monoenergetic proton accretion onto neutron star surfaces. But they did not consider thermal ion distributions applicable to more complex accretion models such as those above. Dahlbacka, Chapline, and Weaver (1974) have calculated high energy $\gamma$-ray emission from decay of pions produced by nuclear interaction of such ions. But this emission, which peaks at $\sim 20 \mathrm{MeV}$ and has a full width at half-maximum (FWHM) $\sim 80 \mathrm{MeV}$, appears to be significant only at the very highest temperatures $\geq 10^{12} \mathrm{~K}$. As we shall show, significant nuclear line emission can be produced at much lower ion temperatures of $10^{10}$ to $10^{11} \mathrm{~K}$ because of the lower threshold energies of the excitation reactions.

We calculate, as a function of ion temperature, the emissivity of the $\gamma$-ray line at $4.438 \mathrm{MeV}$ from deexcitation of the first excited nuclear state of ${ }^{12} \mathrm{C}$. We then consider the detectability of this line above the continuum background and whether the line may already have been observed in the 1972 April $27 \gamma$-ray burst spectrum, measured by Metzger et al. (1974) on A pollo 16.

Both observations (Chupp, Forrest, and Suri 1975; Haymes et al. 1975) and theoretical studies (Ramaty, Kozlovsky, and Lingenfelter 1975; Lingenfelter and Ramaty 1976) of nuclear $\gamma$-ray line emission in astrophysical sites, such as solar flares and the interstellar

* This research was supported in part by National Science Foundation grant AST 76-08178. medium, show that for relative abundances similar to that of the Sun (Cameron, 1973) the most easily detected nuclear de-excitation line is that at $4.438 \mathrm{MeV}$ from ${ }^{12} \mathrm{C}^{*}$. Emission lines at 0.847 and $1.238 \mathrm{MeV}$ from ${ }^{56} \mathrm{Fe}$, $1.369 \mathrm{MeV}$ from ${ }^{24} \mathrm{Mg}, 1.634 \mathrm{MeV}$ from ${ }^{20} \mathrm{Ne}, 1.779$ $\mathrm{MeV}$ from ${ }^{28} \mathrm{Si}, 2.313 \mathrm{MeV}$ from ${ }^{14} \mathrm{~N}$, and $6.129 \mathrm{MeV}$ from ${ }^{16} \mathrm{O}$, though less intense, may also be detectable. In this Letter, however, we shall calculate only the emissivity of the $4.438 \mathrm{MeV}$ line from ${ }^{12} \mathrm{C}^{*}$.

The ${ }^{12} \mathrm{C}^{*}$ production rate and hence the volume emissivity of the $4.438 \mathrm{MeV}$ line from de-excitation of ${ }^{12} \mathrm{C}^{*}$ $\left(\tau_{1 / 2}=5.6 \times 10^{-14} \mathrm{~s}\right)$ excited by thermonuclear interactions in a hot Maxwellian plasma can be written (e.g., Clayton 1968):

$$
\begin{aligned}
Q= & q n^{2}=\Sigma_{i} \Sigma_{j} 4 n^{2} a_{i} a_{j}(2 \pi m)^{-1 / 2}(k T)^{-3 / 2} \mu^{-1 / 2} \\
& \times \int_{0}^{\infty} d E_{i j} E_{i j} \sigma_{i j}\left(E_{i j}\right) \exp \left[-E_{i j} / k T\right]
\end{aligned}
$$

where $n$ is the number density of hydrogen ions; $a_{i}$ and $a_{j}$ are the relative abundances of the interacting nuclei, in this case $i$ is $\mathrm{H}$ and $\mathrm{He}$ and $j$ is $\mathrm{C}$ and $\mathrm{O} ; m$ is the proton mass, $T$ is the ion temperature; $E_{i j}$ is the relative kinetic energy of the $i$ th nuclei with respect to the $j$ th; $\sigma_{i j}\left(E_{i j}\right)$ is the cross section for exciting ${ }^{12} \mathrm{C}$; and $\mu \equiv$ $A_{i} A_{j}\left(A_{i}+A_{j}\right)^{-1}$, where $A$ is the atomic mass number. We calculate $q$ for $a_{\mathrm{H}}=1, a_{\mathrm{He}}=0.07, a_{\mathrm{C}}=3.7 \times 10^{-4}$, and $a_{0}=6.7^{\circ} \times 10^{-4}$ from Cameron (1973) and excitation cross sections for the interactions ${ }^{12} \mathrm{C}\left(\mathrm{H}, \mathrm{H}^{\prime}\right)^{12} \mathrm{C}^{*}$, ${ }^{12} \mathrm{C}\left(\mathrm{He}, \mathrm{He}^{\prime}\right)^{12} \mathrm{C}^{*},{ }^{16} \mathrm{O}(\mathrm{H}, \mathrm{H} \mathrm{He})^{12} \mathrm{C}^{*}$, and ${ }^{16} \mathrm{O}(\mathrm{He}$, $2 \mathrm{He})^{12} \mathrm{C}^{*}$, tabulated in Ramaty, Kozlovsky, and Lingenfelter (1975). The resulting $4.438 \mathrm{MeV}$ line emissivity $q$ is shown in Figure 1 as a function of ion temperature. Interactions between $\mathrm{H}$ and $\mathrm{C}$ cause most of the emission. As can be seen, the emissivity rises rapidly to a broad maximum of $\sim 5 \times 10^{-19} \gamma \mathrm{cm}^{-3} \mathrm{~s}^{-1} n^{-2}$ at about $10^{11} \mathrm{~K}$.

The detectability of this $4.438 \mathrm{MeV}$ emission depends on the line width and on the relative intensity of other 


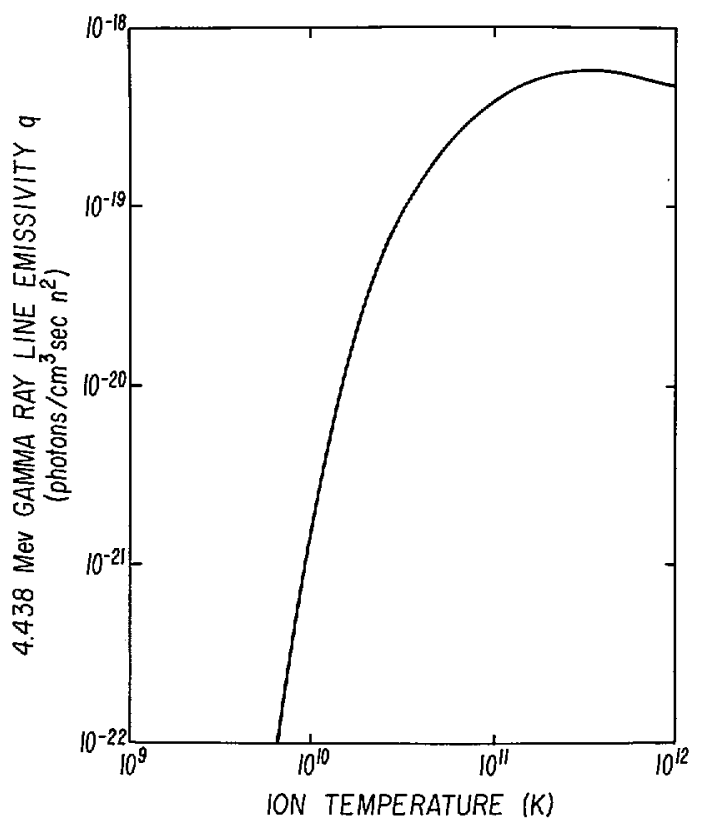

FIG. 1.-Emissivity of the $4.438 \mathrm{MeV} \gamma$-ray line from de-excitation of ${ }^{12} \mathrm{C}^{*}$ produced in thermonuclear interactions as a function of ion temperature.

emission processes, particularly electron bremsstrahlung and Compton scattering, which constitute the background continuum. The line width depends on the velocity distribution of the excited ${ }^{12} \mathrm{C}^{*}$. This is essentially the same as that of unexcited ${ }^{12} \mathrm{C}$, since the average kinetic energy exchanged in excitation interactions between $\mathrm{H}$ and $\mathrm{C}$ nuclei is less than $0.08 k T$. Thus, assuming a Maxwellian distribution, the line full width at half-maximum in $\mathrm{MeV}$ (FWHM) $\approx 9 \times 10^{-7} T^{1 / 2}(\mathrm{~K})$, which gives FWHM of 0.1 to $0.3 \mathrm{MeV}$ for $T$ between $10^{10}$ and $10^{11} \mathrm{~K}$. Therefore because of thermal broadening the differential emissivity of the line ( $q /$ FWHM) reaches a broad maximum of $\sim 10^{-18} \gamma \mathrm{cm}^{-3} \mathrm{~s}^{-1} \mathrm{MeV}^{-1}$ $n^{-2}$ starting at a temperature of $\sim 3 \times 10^{10} \mathrm{~K}$.

In addition, emission lines from the vicinity of highly condensed objects are gravitationally redshifted and also gravitationally broadened, if the gravitational potential varies significantly over the emission volume. For example, in the Shapiro and Salpeter (1975) model of accretion onto neutron stars, the bulk of the emission takes place very near the star surface. The $4.438 \mathrm{MeV}$ line would thus be redshifted anywhere from $0.1 \mathrm{MeV}$ for a neutron star of $0.2 M_{\odot}$ to as much as $\sim 2 \mathrm{MeV}$ or more for masses $\gtrsim 1 M_{\odot}$ (Börner and Cohen 1973). Since the emission region is relatively thin compared to the neutron star radius $(\sim 10 \%)$, the gravitational broadening of the line would likewise be only a fraction $(\sim 10 \%)$ of the redshift, though it still could be comparable to the thermal broadening.

In the Shapiro, Lightman, and Eardley (1976) model of an accretion disk around a black hole, on the other hand, the emission volume is much thicker than $G M / c^{2}$, so that the gravitational broadening exceeds the mean redshift. The same would, of course, be true for an accretion disk around a neutron star. They calculate an inner radius of the high-temperature ion emission region at $6 G M / c^{2}$ and an outer of $28 G M / c^{2}$, so that in the weak field approximation where $Z=G M / c^{2} r$ the 4.438 line would be gravitationally redshifted by an average $\bar{Z}$ of $\sim 0.1$ to $\sim 3.99 \mathrm{MeV}$ and broadened by $\Delta \bar{Z} \approx 0.13$ to $\mathrm{FWHM}=0.58 \mathrm{MeV}$. Unlike thermal broadening, however, gravitational broadening is not symmetrical but essentially reflects the radial dependence of $n^{2}$. Highresolution measurements of line profiles could therefore provide information on the spatial distribution of the ion density in the accretion disk.

Because of the large thermal and gravitational line width as well as the gravitational redshift, resonant scattering of the resultant $\gamma$-ray line emission should not be significant.

It should be noted in passing that since the thermal energy is derived from the gravitational energy, the thermal broadening and average gravitational redshift can be crudely related. In particular $k T<G M m / \bar{r} \approx$ $\bar{Z} m c^{2}$, so that for the $4.438 \mathrm{MeV}$ line FWHM $<10 \bar{Z}^{1 / 2}$ $\mathrm{MeV}$. The specific cases above, of course, satisfy this condition.

Observation of the $4.438 \mathrm{MeV}$ line requires that its differential emissivity $q n^{2} / \mathrm{FWHM}$ be at least comparable to that of the continuum at the line emission energy. Depending on the details of the accretion process the continuum emission at the line energy could be primarily produced either by thermal bremsstrahlung or by Compton scattering. If Compton scattering of lowenergy photons by near relativistic electrons is an important source of the continuum, as in the models discussed above, the differential emissivity of the line would exceed the calculated continuum if $n_{\mathrm{C}} / n_{e} \gtrsim 3 \times$ $10^{-2}$. This is roughly two orders of magnitude above solar values, but could easily be obtained with accretion of cometary material, as has been suggested (Harwit and Salpeter 1973) as a possible cause of $\gamma$-ray bursts. If, on the other hand, the background continuum is thermal electron bremsstrahlung, then the differential emissivity of the $4.438 \mathrm{MeV}$ line exceeds that of the continuum if $T_{\text {ion }}>10^{10} \mathrm{~K}>T_{e}$. This condition is not strongly dependent on the assumed composition.

The detectability of nuclear line emission above either a Compton-scattered or a thermal bremsstrahlung continuum could be significantly enhanced by the existence of magnetic fields in the emission region which increase the energy loss rate of the high-energy electrons, and thus decrease the high-energy continuum emission relative to the line emission. However, if the magnetic field exceeds $10^{12}$ gauss as may happen at the surface of relatively young $\left(<10^{7} \mathrm{yr}\right)$ neutron stars, then the 4.438 $\mathrm{MeV}$ line would be greatly attenuated by pair production in such fields (e.g., Erber 1966). Obviously, more detailed calculations of line emission need to be made in conjunction with specific continuum emission models. But even the crude estimates made here clearly suggest that nuclear lines may be observable with future detectors which extend measurements of X-ray source spectra up to several $\mathrm{MeV}$.

Finally, we consider whether the $4.438 \mathrm{MeV}$ line 
IN. might already have been observed in the spectrum of I the 1972 April $27 \gamma$-ray burst measured (Metzger et al. 1974) on A pollo 16. The energy spectrum of this burst , shows an enhanced emission at about $4 \mathrm{MeV}$, which it is in tempting to attribute to broadened and perhaps red1-I shifted line emission at $4.438 \mathrm{MeV}$. But the measured intensity in this energy range is unfortunately not high enough to permit resolution of such a line with any statistical significance (Metzger 1977, private communication). Previously Metzger et al. (1974) concluded that the enhancement could not be $4.438 \mathrm{MeV}$ line emission because it was too broad, but the expected widths of nuclear lines from accreting matter around neutron stars and black holes, discussed above, no longer make such a width unreasonable.

In conclusion, we have shown that detectable nuclear $4.438 \mathrm{MeV}$ line emission should be observable from
${ }^{12} \mathrm{C}^{*}$ excited by thermonuclear interactions in accreting matter around neutron stars and black holes. Future observations of both continuous and transient $\mathrm{X}$ - and $\gamma$-ray source spectra above $\sim 0.5 \mathrm{MeV}$ with an energy resolution of at least a few percent, should permit detection of the $4.438 \mathrm{MeV}$ and perhaps other nuclear lines. Moreover, the determination of the broadening and redshift of such lines could provide unique information on the ion temperature and the radial density distribution of matter accreting on neutron stars and black holes.

We wish to thank Dr. Albert E. Metzger of the Jet Propulsion Laboratory for making available raw data on the $\gamma$-ray burst spectrum from $A$ pollo 16 , and Dr. Reuven Ramaty of NASA Goddard Space Flight Center for valuable comments.

\section{REFERENCES}

Börner, G., and Cohen, J. M. 1973, Ap. J., 185, 959.

Cameron, A. G. W. 1973, in Explosive Nucleosynthesis, ed. D. S. Schramm and W. D. Arnett (Austin: University of Texas Press), p. 3.

Chupp, E. L., Forrest, D. J., and Suri, A. N. 1975, in IAU Symposium No. 68, Solar Gamma-, $X$-, and EUV Radiation, ed. S. R. Kane (Dordrecht: Reidel), p. 341.

Clayton, D. D. 1968, Principles of Stellar Evolution and Nucleosynthesis (New York: McGraw-Hill).

Dahlbacka, G. H., Chapline, G. F., and Weaver, T. A. 1974, Nature, 250, 36.

Erber, T. 1966. Rev. Mod. Phys., 38, 626.

Harwit, M., and Salpeter, E. E. 1973, A p. J. (Letters), 186, L37.
Haymes, R. C., Walraven, G. D., Meegan, C. A., Hall, R. D., Djuth, F. T., and Shelton, D. G. 1975, Ap. J., 201, 593.

Lingenfelter, R. E., and Ramaty, R. 1976, in The Structure and Content of the Galaxy and Galactic Cosmic Rays, ed. C. E. Fichtel and F. W. Stecker (NASA X-662-76-154), p. 264.

Metzger, A. E., Parker, R. H., Gilman, D., Peterson, L. E., and Trombka, J. I. 1974, Ap. J., (Letters) 194, L19.

Ramaty, R., Börner, G., and Cohen, J. M. 1973, Ap. J., 181, 891.

Ramaty, R., Kozlovsky, B., and Lingenfelter, R. E. 1975, Space Sci. Rev., 23, 198.

Shapiro, S. L., Lightman, A. P., and Eardley, D. M. 1976, Ap.J., $204,187$.

Shapiro, S. L., and Salpeter, E. E. 1975, Ap.J., 198, 671.

J. C. Hrgdon: Cosmic Ray Working Group, Huygens Laboratory, Leiden, The Netherlands

R. E. Lingenfelter: University of California, Department of Astronomy, Los Angeles, CA 90024 
